Proc. Indian Acad. Sci. (Math. Sci.), Vol. 94. No. 1, September 1985, pp. 27-42.

(C) Printed in India.

\title{
On the propagation of a multi-dimensional shock of arbitrary strength
}

\author{
R SRINIVASAN and PHOOLAN PRASAD \\ Department of Applied Mathematics, Indian Institute of Science, Bangalore 560012, India
}

MS received 25 February 1985

\begin{abstract}
In this paper the kinematics of a curved shock of arbitrary strength has been discussed using the theory of generalised functions. This is the extension of Moslov's work where he has considered isentropic flow even across the shock. The condition for a nontrivial jump in the flow variables gives the shock manifold equation (SME). An equation for the rate of change of shock strength along the shock rays (defined as the characteristics of the SME) has been obtained. This exact result is then compared with the approximate result of shock dynamics derived by Whitham. The comparison shows that the approximate equations of shock dynamics deviate considerably from the exact equations derived here. In the last section we have derived the conservation form of our shock dynamic equations. These conservation forms would be very useful in numerical computations as it would allow us to derive difference schemes for which it would not be necessary to fit the shock-shock explicitly.
\end{abstract}

Keywords. Nonlinear waves; shock dynamics; multi-dimensional shock propagation.

\section{Introduction}

It is now about three decades since serious attempts were made to solve the problem of the propagation of a shock of arbitrary shape and arbitrary strength. The main aim was to device a method to calculate the successive positions of a curved shock without going through the task of the calculation of the flow behind the shock. Whitham $[7,8]$ presented one such method, now known as shock dynamics, and showed that the motion of a curved shock depends on the initial shape and the distribution of the shock strength on the initial shock surface. The simplicity and elegance of Whitham's shock dynamics results from the fact that, in this theory, the influence of the waves which catch up with the shock from behind is accounted only approximately [5]. A precise and mathematically satisfying theory on the kinematics of a curved shock was first available in English in 1980 by the translation of the work of the Russian mathematician Maslov [2] who derived a first order partial differential equation satisfied by the shock manifold in $\left(x_{\alpha}, t\right)$-space and then derived an infinite system of compatibility conditions on this manifold. These compatibility conditions are in the form of transport equations along shock rays. The partial differential equation for the shock (called shock manifold equation or SME) is not unique but it has been shown [3] that two different SME $s$ give the same set of shock rays starting from a given initial shock surface. Since Maslov was interested in establishing certain results for a weak shock, he considered the motion of an isentropic gas and thus paid no attention to the energy equation.

In this paper we consider the motion of a shock in an ideal gas with constant specific 
heats with all the three conservation laws, namely the conservation laws representing balance of mass, momentum and energy. We use Maslov's method to derive the SME and the transport equation along a shock ray. Then we use our exact equations to discuss the correct form of the Whitham's shock dynamic equation. We show that shock dynamics not only incorrectly accounts for the effect of the waves which catch up with the shock from behind but the $\mathrm{CCW}$ approximation in the shock dynamics leads to a term whose coefficient differs significantly from the exact coefficient which we get using Maslov's method. Thus this paper shows the mathematical elegance of Maslov's method-the correct form of shock dynamics" can be obtained by a systematic mathematical analysis without any assumption or approximation. Since Maslov's method is not widely known, we shall briefly indicate in the next section some of his important results using the theory of distribution.

\section{Maslov's lemma on a conservation law}

The conservation laws representing the three-dimensional motion of an ideal gas with constant specific heats are

$$
\begin{aligned}
& \frac{\partial \rho}{\partial t}+\langle\nabla, \rho u\rangle=0 \\
& \frac{\partial\left(\rho u^{i}\right)}{\partial t}+\left\langle\nabla, \rho u u^{i}\right\rangle+\frac{\partial p}{\partial x_{i}}=0 \quad(i=1,2,3)
\end{aligned}
$$

and

$$
\frac{\partial}{\partial t}\left\{\frac{1}{2} \rho\langle u, u\rangle+\frac{p}{(\gamma-1)}\right\}+\left\langle\nabla, \frac{1}{2} \rho\langle u, u\rangle u+\frac{\gamma}{(\gamma-1)} p u\right\rangle=0
$$

where $\rho, u=\left(u^{1}, u^{2}, u^{3}\right), p, \gamma$ are mass density, particle velocity, gas pressure, ratio of the specific heats respectively, $\langle$,$\rangle represents scalar product and$

$$
\nabla=\left(\frac{\partial}{\partial x_{1}}, \frac{\hat{c}}{\partial x_{2}}, \frac{\partial}{\partial x_{3}}\right)
$$

is gradient in $R^{3}$.

Consider a piecewise smooth solution of these equations suffering a discontinuity of first kind on a smooth three dimensional surface $\Omega$ in $R^{4}$. Let $\Omega$ be represented by $s\left(x_{1}\right.$, $\left.x_{2}, x_{3}, t\right)=0$. We choose the function $s$ so that $|\nabla s|_{\Omega}=1$. At any fixed time $t, s(x, t)=0$ represents a smooth two-dimensional surface $\Omega_{t}$ in $\left(x_{1}, x_{2}, x_{3}\right)$ space. $\Omega_{t}$ is then a shock surface and we call $\Omega$ shock manifold. The piecewise smooth solution of (1)-(3) can be represented in the form

$$
\left[\begin{array}{l}
\rho(x, t) \\
u(x, t) \\
p(x, t)
\end{array}\right]=\left[\begin{array}{l}
\rho_{0}(x, t) \\
u_{0}(x, t) \\
p_{0}(x, t)
\end{array}\right]+H(s(x, t))\left[\begin{array}{l}
\rho_{1}(x, t) \\
u_{1}(x, t) \\
p_{1}(x, t)
\end{array}\right],
$$

where $\rho_{i}, u_{i}, p_{i}(i=0,1)$ are $C^{x}\left(R^{4}\right)$ functions and $H(\tau)$ is the Heaviside unit function defined by

$$
H(\tau)=\begin{array}{ll}
1, & \tau>0 \\
0, & \tau \leqslant 0
\end{array}
$$


Assuming that $\left(\rho_{0}, u_{0}, p_{0}\right)$ represents the known state ahead of the shock (the domain where $s<0)$ the problem is to find the successive positions of $\Omega_{1}$ and the state $\left(\rho_{0}+\rho_{1}\right.$, $u_{0}+u_{1}, p_{0}+p_{1}$ ) behind $\Omega_{1}$ satisfying some initial and boundary conditions.

Let $R_{\Omega}$ be the set of functions $g$ of the form $g(x, t)=g_{0}(x, t)+H(s) g_{1}(x, t)$, where $g_{0}(x, t)$ and $g_{1}(x, t) \in C^{\infty}\left(R^{4}\right) . R_{\Omega}$ is closed with respect to the operations of addition, subtraction and multiplication. Therefore, all the combinations of $\rho, u, p$ which appear under differentiation operators in (1)-(3) are also elements of $\boldsymbol{R}_{\mathbf{g}}$.

To a piecewise smooth function $g \in \boldsymbol{R}_{\Omega}$, we associate a set of functions defined on the surface of discontinuity $\Omega$ by

$$
\left.g_{i j}\right|_{\Omega}=\left.(\nabla s, \nabla)^{j} g_{i}\right|_{\Omega}, \quad i=0,1 ; j=0,1,2, \ldots
$$

These functions can be interpreted as successive derivatives of $g_{i}(i=0,1)$ along the normal to the shock front $\Omega_{t}$ at any fixed time $t$. We extend any function $\left.g_{i j}\right|_{\Omega}$ defined on $\Omega$ in a smooth manner to the whole of $R^{4}$ so that the extended function $g_{i j}$ is constant along the trajectories of the field $(\nabla s, \nabla)$ i.e., $\left.\left\langle\nabla s, \nabla_{t x}\right\rangle g_{i j}\right|_{\Omega}=0$. Then at any instant $t$, the extended function $g_{i j}$ is constant along a normal to $\Omega_{t}$. We note that $\rho_{00}$ (or $\rho_{10}$ ) need not be equal to $\rho_{0}$ (or $\rho_{1}$ ) in $R^{4}$ except on $\Omega$.

Let $\nabla_{t x}=(\partial / \partial t, \nabla)$ be the four-dimensional gradient in $R^{4}$. $\nabla$ is gradient in $\left(x_{1}, x_{2}\right.$, $x_{3}$ )-space. However, whenever a scalar product of $\nabla$ with an element of $R^{4}$ is taken, it is understood that

$$
\nabla=\left(0, \frac{\partial}{\partial x_{1}}, \frac{\partial}{\partial x_{2}}, \frac{\partial}{\partial x_{3}}\right)
$$

Then for $g \in R_{\Omega}$ and $e=\left(e^{1}, e^{2}, e^{3}, e^{4}\right) \in R^{4}$

$$
\left.\left\langle e-\nabla s\langle\nabla s, e\rangle, \nabla_{t x}\right\rangle g_{i j}\right|_{\Omega}=\left.\left\langle e, \nabla_{t x}\right\rangle g_{i j}\right|_{\Omega},
$$

since $\left.\left\langle\nabla s, \nabla_{t x}\right\rangle g_{i j}\right|_{\Omega}=\left.\langle\nabla s, \nabla\rangle g_{i j}\right|_{\Omega}=0$.

Let the class of generalised functions which are the results of differentiating functions of class $R_{\Omega}$ along some direction $e=\left(e^{1}, e^{2}, e^{3}, e^{4}\right)$ be denoted by $D_{e, \Omega}$. Let $g \in R_{\Omega}$ and $h(x, t)=\left\langle e, \nabla_{t x}\right\rangle g(x, t)$. Then $h \in D_{e . \Omega}$. To $h$ we assign generalised set of functions $\left.h_{i j}\right|_{\Omega}, i=0,1 ; j=-1,0,1, \ldots$, defined on $\Omega$, by

$$
\begin{aligned}
\left.h_{1,-1}(x, t)\right|_{\Omega}= & \left.\left\{g_{10}\left\langle e, \nabla_{t x}\right\rangle s(x, t)\right\}\right|_{\Omega}, \\
\left.h_{i j}(x, t)\right|_{\Omega}= & \left.\left\{g_{i, j+1}\left\langle e, \nabla_{t x}\right\rangle s(x, t)\right\}\right|_{\Omega} \\
& +\left.\left\{\left\langle e-\nabla s\langle e, \nabla s\rangle, \nabla_{t x}\right\rangle g_{i j}\right\}\right|_{\Omega} \\
i= & 0,1 ; j=0,1, \ldots
\end{aligned}
$$

Consider the class of generalised functions $D_{\Omega}$ representable as a finite sum of functions belonging to various sets $D_{e, \Omega}, e \in R^{4}$. Then if $h(x, t) \in D_{\Omega}$, there exists an $m$ $>0$, vectors $e_{1}, e_{2}, \ldots, e_{m}$ and a set of functions $h^{1} \in D_{e_{1}, \Omega}, \ldots, h^{m} \in D_{e_{m}, \Omega}$ such that

$$
h(x, t)=\sum_{i=1}^{m} h^{i}(x, t) \text {. }
$$

To the generalised function $h(x, t) \in D_{\Omega}$ we assign the generalised set $h_{i j}(x, t) ; i=0,1$, $j=-1,0,1,2, \ldots$ in the following manner

$$
h_{i j}(x, t)=\sum_{k=1}^{m} h_{i j}^{k}(x, t)
$$


where $h_{i j}^{k}$ is the sequence associated with $h^{i} \in D_{e_{i, \Omega}}$.

We notice that for a solution of the form (4) the left side of the conservation laws (1)-(3) are actually the elements $h \in D_{\Omega}$. We next state the lemma which plays an important role in Maslov's method.

Lemma. Let $g^{1}, \ldots, g^{m}$ be piecewise-smooth functions belonging to the class $R_{\Omega}$ of functions suffering discontinuity on the same smooth surface $\Omega$ and let $e^{1}, \ldots, e^{m}$ be a collection of vectors in $R^{4}$, if

$$
\sum_{i=1}^{m}\left\langle e^{i}, \nabla_{t x} g^{i}\right\rangle=0
$$

then all terms of the series $\left\{h_{i j}(x, t)\right\}, i=0,1 ; j=-1,0,1,2, \ldots$ defined by equations (10) vanish on the surface $\Omega$.

For a proof of the lemma, reference may be made to Maslov's paper.

\section{The SME and the Rankine-Hugoniot conditions}

Using Maslov's lemma to the solution (4) of the system of conservation laws (1)-(3), we get the following expression for the column matrix $h_{1},-1$

$$
h_{1,-1}(x, t)=\left[\begin{array}{l}
\rho_{10} \frac{\partial s}{\partial t}+\left\langle\nabla s,\left(\rho_{00}+\rho_{10}\right)\left(u_{00}+u_{10}\right)-\rho_{00} u_{00}\right\rangle \\
\left\{\left(\rho_{00}+\rho_{10}\right)\left(u_{00}^{i}+u_{10}^{i}\right)-\rho_{00} u_{00}^{i}\right\} \frac{\partial s}{\partial t} \\
+\left(\rho_{00}+\rho_{10}\right)\left(u_{00}^{i}+u_{10}^{i}\right)\left\langle\nabla s, u_{00}+u_{10}\right\rangle \\
-\rho_{00} u_{00}^{i}\left\langle\nabla s, u_{00}\right\rangle+p_{10} \frac{\partial s}{\partial x_{i}} \\
{\left[\frac{1}{2}\left(\rho_{00}+\rho_{10}\right)\left\langle u_{00}+u_{10}, u_{00}+u_{10}\right\rangle\right.} \\
\left.-\frac{1}{2} \rho_{00}\left\langle u_{00}, u_{00}\right\rangle\right] \frac{\partial s}{\partial t}+\frac{1}{(\gamma-1)} p_{10} \frac{\partial s}{\partial t} \\
+\frac{1}{2}\left(\rho_{00}+\rho_{10}\right)\left\langle u_{00}+u_{10}, u_{00}+u_{10}\right\rangle\left\langle\nabla s, u_{00}+u_{10}\right\rangle \\
-\frac{1}{2} \rho_{00}\left\langle u_{00}, u_{00}\right\rangle\left\langle\nabla s, u_{00}\right\rangle \\
+\frac{\gamma}{(\gamma-1)}\left\{\left(p_{00}+p_{10}\right)\left\langle\nabla s, u_{00}+u_{10}\right\rangle-p_{00}\left\langle\nabla s, u_{00}\right\rangle\right\}
\end{array}\right]=0 .
$$

These are the usual jump conditions across a shock surface. Following the procedure followed by Prasad [3], we deduce the shock manifold equation (SME) in the form

$$
\frac{\partial s}{\partial t}+\left\langle u_{00}, \nabla s\right\rangle+C|\nabla s|=0
$$


where

$$
C^{2}=\frac{\rho_{00}+\rho_{10}}{\rho_{00}} \frac{p_{10}}{\rho_{10}}=\frac{(1+\mu)}{\mu \rho_{0}} p_{10}, \mu=\frac{\rho_{1}}{\rho_{0}} .
$$

The jump conditions across $\Omega$ are given by

$$
\left.u_{10}\right|_{\Omega}=-\left.\xi\left(V_{10} p_{10}\right)^{1 / 2}\right|_{\Omega} ;\left.\frac{p_{00}+p_{10}}{p_{00}}\right|_{\Omega}=\left.\frac{\frac{(\gamma+1)}{(\gamma-1)}(1+\mu)-1}{\frac{(\gamma+1)}{(\gamma-1)}-(1+\mu)}\right|_{\Omega}
$$

where $\xi=\frac{\nabla s}{|\nabla s|}, V_{10}=\frac{1}{\rho_{00}}-\frac{1}{\rho_{00}+\rho_{10}}$

$$
\left.C\right|_{\Omega}=-\left[2\left(\frac{\gamma p_{0}}{\rho_{0}}\right) \frac{(1+\mu)}{\{2+\mu(1-\gamma)\}}\right]^{1 / 2}
$$

and

$$
\mu=\rho_{1} / \rho_{0}
$$

represents the excess density ratio across the shock. $a_{0}$ is the local sound speed in the regions ahead of the shock.

The characteristics of the SME define the shock rays and are given by (we write here this result for a particular case of two spatial variables since we shall directly use it in the next section, $x, y$ are spatial coordinates and $\Theta$ is the angle which the normal to the shock front makes with the $x$-axis)

$$
\frac{\mathrm{d} x_{1}}{\mathrm{~d} \tau}=u_{00}^{1}+C \cos \Theta, \frac{\mathrm{d} x_{2}}{\mathrm{~d} \tau}=u_{00}^{2}+C \sin \Theta,
$$

and

$$
\frac{\mathrm{d} \Theta}{\mathrm{d} \tau}=\left(\sin \Theta \frac{\partial C}{\partial x_{1}}-\cos \Theta \frac{\partial C}{\partial x_{2}}\right)=\frac{\partial C}{\partial \eta},
$$

where $\partial / \partial \eta$ is tangential derivative along the shock curve $\Omega_{t}$ and $d / d \tau$ represents the operator for the temporal derivative of a quantity along a shock ray.

\section{The second compatibility condition on the shock manifold}

Maslov gives a method for the derivation of an infinite set of compatibility conditions on the shock manifold. The first group of such relations in this set is the RankineHugoniot condition (14)-(15). The second group, namely the jump conditions on the first derivatives, consists of an expression for the rate of change of $\mu=\rho_{1} / \rho_{0}$ along a shock ray in terms of a tangential derivative of $\Theta$ in $\Omega_{t}$ and a normal derivative of $\mu$ and also some algebraic relations amongst the normal derivatives of the dependent variables. Our aim in this section is to derive one of the compatibility conditions in this group - namely the above mentioned expression for the rate of change of $\mu$ along the shock ray. For the discussion in the next section, we need only this part of the compatibility condition.

From Maslov's lemma for a solution (4) of (1) to (3) it follows that all the components 
of the vector $h_{10}$ vanish on $\Omega$. This gives

$$
\begin{aligned}
& \left.\left\{\rho_{11} K_{l}+\left(\rho_{00}+\rho_{10}\right)\left\langle\nabla s, u_{11}\right\rangle+z\right\}\right|_{\Omega}=0, \\
& \rho_{11} K_{l}\left(u_{00}^{i}+u_{10}^{i}\right)+\left(\rho_{00}+\rho_{10}\right)\left(u_{00}^{i}+u_{10}^{i}\right)\left\langle\nabla s, u_{11}\right\rangle \\
& +\left(\rho_{00}+\rho_{10}\right) u_{11}+p_{11} \frac{\partial s}{\partial x_{i}}+\left.\omega^{i}\right|_{\Omega}=0,
\end{aligned}
$$

and

$$
\begin{aligned}
& \frac{1}{2} \rho_{11}\left\langle u_{00}+u_{10}, u_{00}+u_{10}\right\rangle K_{l}+\left(\rho_{00}+\rho_{10}\right) \\
& \left\langle u_{00}+u_{10}, u_{11}\right\rangle K_{l}+\frac{1}{2}\left(\rho_{00}+\rho_{10}\right)\left\langle u_{00}+u_{10}\right. \\
& \left.u_{00}+u_{10}\right\rangle\left\langle\nabla s, u_{11}\right\rangle+\frac{1}{(\gamma-1)} p_{11} \frac{\partial s}{\partial t} \\
& +\frac{\gamma}{(\gamma-1)}\left[\left(p_{00}+p_{10}\right)\left\langle\nabla s, u_{11}\right\rangle+p_{11}\left\langle\nabla s, u_{00}+u_{10}\right\rangle\right]+\left.M\right|_{\Omega}=0
\end{aligned}
$$

where

$$
\begin{aligned}
K_{l} & =\frac{\partial s}{\partial t}+\left\langle\nabla s, u_{00}+u_{10}\right\rangle, \\
z & =\frac{\partial \rho_{10}}{\partial t}+\left(\rho_{00}+\rho_{10}\right)\left\langle\nabla s, u_{01}\right\rangle+\rho_{01}\left\langle\nabla s, u_{00}\right. \\
& \left.+u_{10}\right\rangle-\left\langle\nabla s, \rho_{00} u_{01}+\rho_{01} u_{00}\right\rangle \\
& +\left\langle\nabla,\left(\rho_{00}+\rho_{10}\right)\left(u_{00}+u_{10}\right)-\rho_{00} u_{00}\right\rangle \\
\omega^{i} & =\frac{\partial s}{\partial t}\left[\left(\rho_{00}+\rho_{10}\right) u_{01}^{i}+\rho_{01}\left(u_{00}^{i}+u_{10}^{i}\right)-\rho_{00} u_{01}^{i}\right. \\
& \left.-\rho_{01} u_{00}^{i}\right]+\frac{\partial}{\partial t}\left[\left(\rho_{00}+\rho_{10}\right)\left(u_{00}^{i}+u_{10}^{i}\right)-\rho_{00} u_{00}^{i}\right] \\
+ & \left(\rho_{00}+\rho_{10}\right)\left(u_{00}^{i}+u_{10}^{i}\right)\left\langle\nabla s, u_{01}\right\rangle+\left(\rho_{00}+\rho_{10}\right) u_{01}^{i}\left\langle\nabla s, u_{00}+u_{10}\right\rangle \\
+ & \rho_{01}\left(u_{00}^{i}+u_{10}^{i}\right)\left\langle\nabla s, u_{00}+u_{10}\right\rangle \\
- & \left\langle\nabla s, \rho_{00} u_{00}^{i} u_{01}+\rho_{00} u_{01}^{i} u_{00}+\rho_{01} u_{00}^{i} u_{00}\right\rangle \\
+ & \left\langle u_{00}+u_{10}, \nabla\right\rangle\left(\rho_{00}+\rho_{10}\right)\left(u_{00}^{i}+u_{10}^{i}\right) \\
+ & \left(\rho_{00}+\rho_{10}\right)\left(u_{00}^{i}+u_{10}^{i}\right)\left\langle\nabla, u_{00}+u_{10}\right\rangle-\rho_{00} u_{00}^{i}\left\langle\nabla, u_{00}\right\rangle \\
& -\left\langle u_{00}, \nabla\right\rangle \rho_{00} u_{00}^{i}+\frac{\partial p_{10}}{\partial x_{i}}, \\
+ & +\frac{\partial}{\partial t}\left[\frac{1}{2}\left(\rho_{00}+\rho_{10}\right)\left\langle u_{00}+u_{10}, u_{00}+u_{10}\right\rangle\left\langle\nabla s, u_{00}+u_{10}\right\rangle\right. \\
M & =\frac{\partial s}{\partial t}\left[\frac{1}{2} \rho_{01}\left\langle u_{00}+u_{10}, u_{00}+u_{10}\right\rangle\right]+\frac{1}{(\gamma-1)} \frac{\partial p_{10}}{\partial t}+\frac{1}{2} \rho_{01}\left\langle u_{00}+u_{10}, u_{00}+u_{10}\right\rangle \\
+ & \left.\left.+\rho_{10}\right)\left\langle u_{00}+u_{10}, u_{01}\right\rangle-\frac{1}{2} \rho_{01}\left\langle u_{00}, u_{00}\right\rangle-\rho_{00}\left\langle u_{00}, u_{01}\right\rangle\right] \\
& \\
- &
\end{aligned}
$$




$$
\begin{aligned}
& \left\langle\nabla s, u_{00}+u_{10}\right\rangle+\frac{1}{2}\left(\rho_{00}+\rho_{10}\right)\left\langle u_{00}+u_{10}, u_{00}+u_{10}\right\rangle\left\langle\nabla s, u_{01}\right\rangle \\
& +\left(\rho_{00}+\rho_{10}\right)\left\langle u_{00}+u_{10}, u_{01}\right\rangle\left\langle\nabla s, u_{00}+u_{10}\right\rangle \\
& -\frac{1}{2} \rho_{00}\left\langle u_{00}, u_{00}\right\rangle\left\langle\nabla s, u_{01}\right\rangle-\frac{1}{2} \rho_{01}\left\langle u_{00}, u_{00}\right\rangle\left\langle\nabla s, u_{00}\right\rangle \\
& -\rho_{00}\left\langle u_{00}, u_{01}\right\rangle\left\langle\nabla s, u_{00}\right\rangle+\frac{1}{2}\left(\rho_{00}+\rho_{10}\right)\left\langle u_{00}+u_{10}, u_{00}+u_{10}\right\rangle \\
& \left\langle\nabla, u_{00}+u_{10}\right\rangle+\left\langle u_{00}+u_{10}, \nabla\right\rangle\left\{\frac{1}{2}\left(\rho_{00}+\rho_{10}\right)\right. \\
& \left.\cdot\left\langle u_{00}+u_{10}, u_{00}+u_{10}\right\rangle\right\}-\frac{1}{2} \rho_{00}\left\langle u_{00}, u_{00}\right\rangle\left\langle\nabla, u_{00}\right\rangle \\
& -\left\langle u_{00}, \nabla\right\rangle \frac{1}{2} \rho_{00}\left\langle u_{00}, u_{00}\right\rangle+\frac{\gamma}{(\gamma-1)}\left[p_{10}\left\langle\nabla s, u_{01}\right\rangle\right. \\
& \left.+p_{01}\left\langle\nabla s, u_{10}\right\rangle+\left\langle\nabla,\left(p_{00}+p_{10}\right)\left(u_{00}+u_{10}\right)-p_{00} u_{00}\right\rangle\right] .
\end{aligned}
$$

Multiplying (19) by $\left(u_{00}+u_{10}\right)$ and subtracting from (20) we get

$$
\left(\rho_{00}+\rho_{10}\right) u_{11} K_{l}+p_{11} \nabla s+\omega-\left(u_{00}+u_{10}\right) z=0 .
$$

Multiplying (19) by $\frac{1}{2}\left\langle u_{00}+u_{10}, u_{00}+u_{10}\right\rangle$ and subtracting from (21) we get

$$
\begin{aligned}
& \left(\rho_{00}+\rho_{10}\right)\left\langle u_{00}+u_{10}, u_{11}\right\rangle K_{l}+\frac{1}{(\gamma-1)} p_{11} \frac{\partial s}{\partial t} \\
& +\frac{\gamma}{(\gamma-1)}\left[\left(p_{00}+p_{10}\right)\left\langle\nabla s, u_{11}\right\rangle+p_{11}\left\langle\nabla s, u_{00}+u_{10}\right\rangle\right] \\
& +M-\frac{1}{2}\left\langle u_{00}+u_{10}, u_{00}+u_{10}\right\rangle z=0 .
\end{aligned}
$$

Multiplying (26) by $\left(u_{00}+u_{10}\right)$ and subtracting from (27) we get

$$
\begin{aligned}
& \frac{1}{(\gamma-1)} p_{11} K_{l}+\frac{\gamma}{(\gamma-1)}\left(p_{00}+p_{10}\right)\left\langle\nabla s, u_{11}\right\rangle \\
& +M-\frac{1}{2}\left\langle u_{00}+u_{10}, u_{00}+u_{10}\right\rangle z-\left\langle\omega, u_{00}+u_{10}\right\rangle \\
& +\left\langle u_{00}+u_{10}, u_{00}+u_{10}\right\rangle z=0 .
\end{aligned}
$$

Multiplying (26) by $\frac{1}{(\gamma-1)} K_{l} \nabla s$ and subtracting from (28) we get

$$
\begin{aligned}
& \alpha\left(\rho_{00}+\rho_{10}\right)\left\langle\nabla s, u_{11}\right\rangle+M-\frac{1}{2}\left\langle u_{00}+u_{10}, u_{00}+u_{10}\right\rangle z \\
& -\left\langle\omega, u_{00}+u_{10}\right\rangle+\left\langle u_{00}+u_{10}, u_{00}+u_{10}\right\rangle z-\frac{1}{(\gamma-1)} K_{l}\langle\nabla s, \omega \\
& \left.-\left(u_{00}+u_{10}\right) z\right\rangle=0
\end{aligned}
$$

where $\alpha$ is defined by (31).

Multiplying (19) by $\alpha$ and subtracting from (29) we get

$$
\begin{aligned}
& M-\frac{1}{2}\left\langle u_{00}+u_{10}, u_{00}+u_{10}\right\rangle z-\left\langle\omega, u_{00}+u_{10}\right\rangle \\
& +z\left\langle u_{00}+u_{10}, u_{00}+u_{10}\right\rangle-\frac{1}{(\gamma-1)} K_{l}\left\langle\omega-\left(u_{00}+u_{10}\right) z, \nabla s\right\rangle \\
& -\alpha z-\alpha \rho_{11} K_{l}=0,
\end{aligned}
$$


where

$$
\alpha=\left\{\frac{\gamma}{(\gamma-1)} \frac{p_{00}+p_{10}}{\rho_{00}+\rho_{10}}-\frac{K_{l}^{2}}{\gamma-1}\right\}=a_{0}^{2} \frac{\mu(1+\gamma)}{(\gamma-1)(1+\mu)\{2+\mu(1-\gamma)\}}
$$

The operator $d / d \tau$ in (17) and (18) gives rate of change along a shock ray i.e., it represents an inner derivative in the shock manifold $\Omega: s(x, t)=0$. Therefore, for a function $\left.g_{i j}\right|_{\Omega}$ defined initially on $\Omega$ and extended as $g_{i j}$ in $R^{4}$ so that it is constant on the trajectories of the field $\langle\nabla s, \nabla\rangle$,

$$
\left.\frac{\mathrm{d}}{\mathrm{d} \tau} g_{i j}\right|_{\mathbf{\Omega}}
$$

can be obtained from $\left.g_{i j}\right|_{\Omega}$ alone. From (14) it follows that $\left.u_{10}\right|_{\Omega}$ is parallel to the vector $\left.\nabla s\right|_{\Omega}$, hence $\left.\left\langle u_{10}, \nabla\right\rangle g_{i j}\right|_{\Omega}=0$. Therefore,

$$
\begin{aligned}
& \left.\left\{\frac{\partial}{\partial t}+\left\langle u_{00}, \nabla\right\rangle\right\} g_{i j}\right|_{\Omega}=\left.\left\{\frac{\partial}{\partial t}+\left\langle u_{00}+u_{10}, \nabla\right\rangle\right\} g_{i j}\right|_{\Omega} \\
& =\left.\left\{\frac{\partial}{\partial t}+\left\langle u_{00}+C \xi, \nabla\right\rangle\right\} g_{i j}\right|_{\Omega}, \xi=\frac{\nabla s}{|\nabla s|} .
\end{aligned}
$$

Using these results, we find the following expressions for $M-\frac{1}{2}<u_{00}+u_{10}, u_{00}+$

$$
\begin{aligned}
& \left.u_{10}\right\rangle z,\left\langle\omega, u_{00}+u_{10}\right\rangle, \text { and } w-\left(u_{00}+u_{10}\right) z \text { on } \Omega: \\
& M-\left.\frac{1}{2}\left\langle u_{00}+u_{10}, u_{00}+u_{10}\right\rangle\right|_{\Omega}= \\
& -C\left[\rho_{01}\left\langle u_{10}, u_{10}\right\rangle+\frac{1}{2} \rho_{01}\left\langle u_{10}, u_{10}\right\rangle+\rho_{10}\left\langle u_{00}+u_{10}, u_{01}\right\rangle\right. \\
& \left.+\rho_{00}\left\langle u_{10}, u_{01}\right\rangle\right]+\frac{\mathrm{d}}{\mathrm{d} \tau}\left[\frac{1}{2}\left(\rho_{00}+\rho_{10}\right)\left\langle u_{00}+u_{10}, u_{00}+u_{10}\right\rangle\right. \\
& \left.-\frac{1}{2} \rho_{00}\left\langle u_{00}, u_{00}\right\rangle\right]-\frac{1}{2}\left\langle u_{00}+u_{10}, u_{00}+u_{10}\right\rangle \frac{\mathrm{d}\left(\rho_{00}+\rho_{10}\right)}{\mathrm{d} \tau} \\
& +\frac{1}{2}\left\langle u_{00}+u_{10}, u_{00}+u_{10}\right\rangle \frac{\mathrm{d} \rho_{00}}{\mathrm{~d} \tau}+\frac{1}{(\gamma-1)} \frac{\mathrm{d} p_{10}}{\mathrm{~d} \tau} \\
& +\left[\left(\rho_{00}+\rho_{10}\right)\left\langle u_{00}+u_{10}, u_{01}\right\rangle+\frac{\gamma p_{01}}{(\gamma-1)}\right]\left\langle\nabla s, u_{10}\right\rangle \\
& +\left[\rho_{00}\left\langle u_{00}, u_{10}\right\rangle+\frac{1}{2} \rho_{00}\left\langle u_{10}, u_{10}\right\rangle+\frac{\gamma p_{10}}{(\gamma-1)}\right]\left\langle\nabla s, u_{01}\right\rangle \\
& -\frac{1}{2} \rho_{00}\left\langle u_{00}, u_{00}\right\rangle\left\langle\nabla, u_{00}\right\rangle+\frac{1}{2}\left\langle u_{00}+u_{10}, u_{00}+u_{10}\right\rangle \rho_{00}\left\langle\nabla, u_{00}\right\rangle \\
& +\frac{\gamma}{(\gamma-1)}\left[\left(p_{00}+p_{10}\right)\left\langle\nabla, u_{00}+u_{10}\right\rangle+\left\langle u_{00}+u_{10}, \nabla\right\rangle p_{10}\right] \\
& \left\langle\omega, u_{00}+u_{10}\right\rangle= \\
& \left\langle u_{00}+u_{10}, \frac{\mathrm{d}}{\mathrm{d} \tau}\left\langle\left(\rho_{00}+\rho_{10}\right)\left(u_{00}+u_{10}\right)-\rho_{00} u_{00}\right\}\right\rangle
\end{aligned}
$$




$$
\begin{aligned}
& +\left(\rho_{00}+\rho_{10}\right)\left\langle u_{00}+u_{10}, u_{00}+u_{10}\right\rangle\left\langle\nabla s, u_{01}\right\rangle \\
& +\rho_{01}\left\langle u_{00}+u_{10}, u_{00}+u_{10}\right\rangle\left\langle\nabla s, u_{10}\right\rangle-\rho_{00}\left\langle u_{00}, u_{00}+u_{10}\right\rangle\left\langle\nabla s, u_{01}\right\rangle \\
& \quad+\left(\rho_{00}+\rho_{10}\right)\left\langle u_{00}+u_{10}, u_{01}\right\rangle\left\langle\nabla s, u_{10}\right\rangle \\
& -C\left[\rho_{10}\left\langle u_{00}+u_{10}, u_{01}\right\rangle+\rho_{01}\left\langle u_{00}+u_{10}, u_{10}\right\rangle\right] \\
& +\left(\rho_{00}+\rho_{10}\right)\left\langle u_{00}+u_{10}, u_{00}+u_{10}\right\rangle\left\langle\nabla, u_{00}+u_{10}\right\rangle \\
& -\rho_{00}\left\langle u_{00}+u_{10}, u_{00}\right\rangle\left\langle\nabla, u_{00}\right\rangle+\left\langle u_{00}+u_{10}, \nabla\right\rangle p_{10}
\end{aligned}
$$

and

$$
\begin{aligned}
& w-\left(u_{00}+u_{10}\right) z= \\
& -C\left[\rho_{10} u_{01}+\rho_{01} u_{10}\right]+\frac{\mathrm{d}}{\mathrm{d} \tau}\left[\left(\rho_{00}+\rho_{10}\right)\left(u_{00}+u_{10}\right)-\rho_{00} u_{00}\right] \\
& -\left(u_{00}+u_{10}\right) \frac{\mathrm{d} \rho_{10}}{\mathrm{~d} \tau}+\left(\rho_{00}+\rho_{10}\right) u_{01}\left\langle\nabla s, u_{10}\right\rangle \\
& +\rho_{00} u_{10}\left\langle\nabla s, u_{01}\right\rangle+\rho_{00} u_{10}\left\langle\nabla, u_{00}\right\rangle+\nabla p_{10} .
\end{aligned}
$$

Substituting these values in (30) we get

$$
\begin{aligned}
& \frac{1}{(\gamma-1)} \frac{\mathrm{d} p_{10}}{\mathrm{~d} \tau}+\frac{C \rho_{0}}{(\gamma-1)}\left\langle\nabla s, \frac{\mathrm{d} u_{10}}{\mathrm{~d} \tau}\right\rangle+\frac{C \rho_{0} \mu}{(\gamma-1)(1+\mu)}\left\langle\nabla s, \frac{\mathrm{d} u_{00}}{\mathrm{~d} \tau}\right\rangle \\
& +\frac{C}{(\gamma-1)(1+\mu)}\left\langle\nabla s, u_{10}\right\rangle \frac{\mathrm{d} \rho_{00}}{\mathrm{~d} \tau}+\rho_{00}\left\langle u_{10}, \frac{\mathrm{d} u_{00}}{\mathrm{~d} \tau}\right\rangle \\
& -\frac{1}{2}\left\langle u_{10}, u_{10}\right\rangle \frac{\mathrm{d} \rho_{00}}{\mathrm{~d} \tau}-\alpha \frac{\mathrm{d} \rho_{10}}{\mathrm{~d} \tau}+\rho_{01}\left[\frac{1}{2} \frac{C^{3} \mu}{(1+\mu)^{2}}-\alpha\left\langle\nabla s, u_{10}\right\rangle\right. \\
& \left.-\frac{C^{3} \mu}{(\gamma-1)(1+\mu)^{2}}\right]+\left\langle\nabla s, u_{01}\right\rangle\left[\frac{-C^{2} \rho_{0} \mu}{(1+\mu)}-\frac{1}{2} \rho_{0} \frac{C^{2} \mu^{2}}{(1+\mu)^{2}}\right. \\
& +\frac{\gamma c^{2} \mu \rho_{0}}{(\gamma-1)(1+\mu)}-\frac{c^{2} \mu \rho_{0}}{(\gamma-1)(1+\mu)}-\alpha \rho_{10}+\frac{c^{2} \mu \rho_{0}}{(\gamma-1)(1+\mu)} \\
& \left.+\frac{C^{2} \mu \rho_{0}}{(\gamma-1)(1+\mu)^{2}}\right]+\frac{\gamma p_{01}}{(\gamma-1)}\left\langle\nabla s, u_{10}\right\rangle \\
& +\left[\frac{\gamma}{(\gamma-1)}\left(p_{00}+p_{10}\right)-\alpha\left(\rho_{00}+\rho_{10}\right)\right]\left\langle\nabla, u_{10}\right\rangle \\
& +\left[\frac{\gamma}{(\gamma-1)} p_{10}+\frac{C^{2} \mu \rho_{0}}{(\gamma-1)(1+\mu)^{2}}-\alpha \rho_{10}-\frac{1}{2}\left\langle u_{10}, u_{10}\right\rangle\right]\left\langle\nabla, u_{00}\right\rangle .
\end{aligned}
$$

Since the operator $\mathrm{d} / \mathrm{d} \tau$ gives an interior derivative in $\Omega$, we note that in the above we can use

$$
\mathrm{d} \rho_{00} / \mathrm{d} \tau=\mathrm{d} \rho_{0} / \mathrm{d} \tau, \quad \frac{\mathrm{d}}{\mathrm{d} \tau}\left(\rho_{00} u_{00}\right)=\frac{\mathrm{d}\left(\rho_{0} u_{0}\right)}{\mathrm{d} \tau}
$$


etc. The set $\left\{\rho_{0}(x, t), u_{0}(x, t), p_{0}(x, t)\right\}$ is a genuine solution of equations (1) to (3), hence

$$
\begin{aligned}
\left.\frac{\mathrm{d} \rho_{00}}{\mathrm{~d} \tau}\right|_{\Omega} & =\left.\frac{\mathrm{d} \rho_{0}}{\mathrm{~d} \tau}\right|_{\Omega}=\left.\left(\frac{\partial \rho_{0}}{\partial t}+\left\langle u_{0}, \nabla\right\rangle \rho_{0}+C\langle\nabla s, \nabla\rangle \rho_{0}\right)\right|_{\Omega} \\
& =-\rho_{0}\left\langle\nabla, u_{0}\right\rangle+\left.C \rho_{01}\right|_{\Omega}
\end{aligned}
$$

Similarly, we get

$$
\left.\frac{\mathrm{d} u_{00}}{\mathrm{~d} \tau}\right|_{\Omega}=\frac{1}{\rho_{0}}\left(C \rho_{0} u_{01}-\nabla p_{0}\right)
$$

and

$$
\rho_{01}=\frac{1}{C}\left(\rho_{0}\left\langle\nabla, u_{0}\right\rangle+\frac{\mathrm{d} \rho_{00}}{\mathrm{~d} \tau}\right) .
$$

We also note

$$
\begin{aligned}
& \left\langle\xi, \frac{\mathrm{d} \xi}{\mathrm{d} \tau}\right\rangle=0,\left.\langle\zeta, L\rangle\right|_{\Omega}=0, \\
& \left.\left\langle\nabla, u_{0}\right\rangle\right|_{\Omega}=\left.\left\langle\nabla, u_{00}\right\rangle\right|_{\Omega}+\left.\left\langle\nabla s, u_{01}\right\rangle\right|_{\Omega},
\end{aligned}
$$

where

$$
L=\nabla-\xi\langle\xi, \nabla\rangle
$$

In terms of the rate of change of the excess density ratio, $\mu=\left(\rho_{1} / \rho_{0}\right)$ across the shock, eliminating $p_{10}$ and $u_{10}$ from (14) and (15) respectively and using (33) to (36) we write (32) in the form

$$
\begin{aligned}
& \left\{\frac{2 C^{2} \rho_{0}}{(\gamma-1)(1+\mu)^{2}}+\frac{2 C \mu \rho_{0}}{(\gamma-1)(1+\mu)} \frac{\partial c}{\partial \mu}-\alpha \rho_{0}\right\} \frac{\mathrm{d} \mu}{\mathrm{d} \tau} \\
& +\left\{\frac{C^{2} \mu}{(\gamma-1)(1+\mu)}+\frac{3 C \mu \rho_{0}}{(\gamma-1)(1+\mu)} \frac{\partial c}{\partial \rho_{0}}-\alpha \mu \frac{(2+\mu)}{(1+\mu)}\right\} \frac{\mathrm{d} \rho_{00}}{\mathrm{~d} \tau} \\
& +\frac{3 C \mu \rho_{0}}{(\gamma-1)(1+\mu)} \frac{\partial C}{\partial p_{0}} \frac{\mathrm{d} p_{00}}{\mathrm{~d} \tau}+\frac{C^{2} \mu \rho_{0}}{(\gamma-1)(1+\mu)^{2}}\langle L, \xi\rangle \\
& +\frac{C^{2} \mu \rho_{0}}{(\gamma-1)(1+\mu)}\left\langle\nabla s, u_{01}\right\rangle+\left[\frac{\gamma}{(\gamma-1)} \frac{C^{2} \mu \rho_{0}}{(1+\mu)}-\right. \\
& \left.-\frac{\alpha \mu \rho_{0}(2+\mu)}{(1+\mu)}\right]\left\langle\nabla, u_{0}\right\rangle+\left.\frac{\alpha C \rho_{11}}{(1+\mu)}\right|_{\Omega}=0 .
\end{aligned}
$$

This is the required compatibility condition giving the rate of change of $\mu$ along a shock ray in terms of the known quantities in the region ahead of the shock, the interior derivatives of unit normal $\zeta$ of the shock front $\Omega_{t}$ and a normal derivative $\rho_{11}$.

\section{Correct form of the shock dynamic equations in two-space dimensions}

In the case of two space variables $x_{1}, x_{2}$, the shock $\Omega_{t}$ at time $t$ will be represented by a curve in $\left(x_{1}, x_{2}\right)$ plane. Let $\Theta$ be the angle which the normal to $\Omega_{t}$ makes with the $x_{1}$-axis. 
Then $\xi=(\cos \Theta, \sin \Theta)$. Assuming the state ahead of the shock to be a constant state and at rest, i.e. $u_{0}^{1}=0, u_{0}^{2}=0$, and using $\langle L, \xi\rangle=\partial \Theta / \partial \eta$ we get from (42)

$$
\frac{\partial \mu}{\partial s_{1}}=B_{1} \frac{\partial \Theta}{\partial \eta}+B_{2} \frac{\partial \mu}{\partial N}
$$

where $\mathrm{d} s_{1}$ is an element of length described in time $\mathrm{d} t$ in $\left(x_{1}, x_{2}\right)$-plane while moving along a shock ray ( $\left.\mathrm{d} s_{1}=C \mathrm{~d} t\right), \mathrm{d} \eta$ is an element of length along the shock curve $\Omega_{t}$ at any time and $\mathrm{d} N$ is an element of length in a direction normal to $\Omega_{t}$ :

$$
\begin{aligned}
& \frac{\partial}{\partial s_{1}}=\frac{1}{C}\left(\frac{\partial}{\partial t}+\left(u_{0}^{1}+C \cos \Theta\right) \frac{\partial}{\partial x_{1}}+\left(u_{0}^{2}+C \sin \Theta\right) \frac{\partial}{\partial x_{2}}\right) \\
& \frac{\partial}{\partial \eta}=\sin \Theta \frac{\partial}{\partial x_{1}}-\cos \Theta \frac{\partial}{\partial x_{2}}
\end{aligned}
$$

and

$$
\frac{\partial}{\partial N}=\cos \Theta \frac{\partial}{\partial x_{1}}+\sin \Theta \frac{\partial}{\partial x_{2}} .
$$

The coefficients $B_{1}$ and $B_{2}$ in (43) are given by

$$
B_{1}=-\frac{2 \mu\{2+\mu(1-\gamma)\}}{8+6 \mu-2 \gamma \mu-\mu^{2}\left(1-\gamma^{2}\right)}
$$

and

$$
B_{2}=-\frac{\mu(1+\gamma)\{2+\mu(1-\gamma)\}}{\left\{8+6 \mu-2 \gamma \mu-\mu^{2}\left(1-\gamma^{2}\right)\right\}(1+\mu)} .
$$

Substituting in (18) the expression for $C$ in terms of $\mu$, we get

$$
\frac{\partial \Theta}{\partial s_{1}}=-\frac{(\gamma+1)}{2(1+\mu)\{2+\mu(1-\gamma)\}} \frac{\partial \mu}{\partial \eta} .
$$

Equations (43) and (49) could be used to calculate the successive positions of a shock front provided the term $\partial \mu / \partial N$ can be estimated in (43). This term can be approximately evaluated for a class of problems when the shock is weak [4]. However, since (43) and (49) are exact equations, it would be interesting to compare these with the corresponding equations of Whitham's shock dynamics $[7,8]$, which even today, is the only successful nonnumerical technique for the determination of the successive positions of a curved shock. We shall first give here Whitham's shock dynamics equations for shock motion in two space dimensions.

Assuming that the state ahead of the shock is at rest the successive positions of the shock and the corresponding family of shock rays form a set of orthogonal curvilinear coordinates. Let $\alpha=a_{0} t=$ constant represent the family of curves giving the successive positions of the shock and $\beta=$ constant represent the family of shock rays. The line elements along the two families of curves are $M \mathrm{~d} \alpha$ and $A \mathrm{~d} \beta$, where $M$ is the shock Mach number and $A$ is an appropriately defined area of an elementary shock ray tube. Considering the changes in the shock ray inclination $\Theta$ and the shock Mach number along the shock curve, we get the following two equations in $\Theta, M$ and $A$ :

$$
\partial \Theta / \partial \alpha=-(1 / A)(\partial M / \partial \beta)
$$


and

$$
\partial A / \partial \alpha=M(\partial \Theta / \partial \beta)
$$

To obtain a determinate system of equations, Whitham assumed that the flow in each ray tube can be treated as one-dimensional, and a functional relation between $A$ and $M$ can be obtained by using the ccw approximation, in the form $A=A_{0} f(M) / f\left(M_{0}\right)$ where $f(M)$ is given by

$$
\begin{aligned}
f(M) & =\exp \left\{-\int \frac{M \lambda(M)}{M^{2}-1} \mathrm{~d} M\right\} \\
\lambda(M) & =\left\{1+\frac{2}{(\gamma+1)} \frac{\left(1-d^{2}\right)}{d}\right\}\left\{1+2 d+\frac{1}{M^{2}}\right\} \\
d^{2} & =\frac{(\gamma-1) M^{2}+2}{2 \gamma M^{2}-(\gamma-1)} .
\end{aligned}
$$

Once $\Theta$ and $M$ are determined as functions of $\alpha, \beta$, the position of the shock at any time $t=\alpha / a_{0}$ can be obtained by integrating the ray equations

$$
\partial x / \partial \alpha=M \cos \Theta, \partial y / \partial \alpha=M \sin \Theta
$$

The operators $\partial / \partial \alpha$ and $\partial / \partial \beta$ give interior derivatives in the shock manifold $\Omega$. In order to compare the compatibility conditions of the shock dynamics with those derived by Maslov's method, we write (50) and (51) in terms of the excess density ratio $\mu$ across the shock and in terms of spatial distances $s$ and $\eta$ related to $\alpha$ and $\beta$ by $\mathrm{d} s_{1}=M \mathrm{~d} \alpha$ and $\mathrm{d} \eta=A \mathrm{~d} \beta$ respectively. The results are

$$
\partial \mu / \partial s_{1}=B_{3}(\partial \Theta / \partial \eta)
$$

and

$$
\frac{\partial \Theta}{\partial s_{1}}=-\frac{(\gamma+1)}{2(1+\mu)\{2+\mu(1-\gamma)\}} \frac{\partial \mu}{\partial \eta}
$$

where

$$
B_{3}=\frac{-2^{3 / 2}\{2+\mu(1-\gamma)\}(1+\mu)[2+(\gamma+1) \mu]^{1 / 2}}{\sqrt{2}\{4+\mu(3-\gamma)\}[2+(\gamma+1) \mu]^{1 / 2}+(\gamma+1) \mu\{4+\mu(3-\gamma)\}+8(1+\mu)} .
$$

We note that (54) of shock dynamics is exactly the same as (49) derived by Maslov's method. However, the second term $B_{2}(\partial \mu / \hat{C} N)$ of (43) is missing in (53) of shock dynamics. This term is of the same order as the first two terms unless the state behind the shock is almost a uniform state. The limiting form of (43) and (49) have been derived for a weak shock by Ramanathan et al [5] by another method. We note that, in this case, i.e. $\mu \ll 1$, both coefficients $B_{1}$ (of Maslov's method) and $B_{3}$ (of shock dynamics) can be approximated by the same value $-\frac{1}{2} \mu$ as derived in [5]. For a shock of arbitrary strength table 1 gives a comparison of the values of $B_{1}$ and $B_{3}$. Unlike the intuitive and approximate deduction of the shock dynamic equations, Maslov's theory gives the exact results by a mathematically elegant method. The authors believe that Maslov's theory will be of great value in the theory of multi-dimensional shock propagation. 
Table 1. Comparison between Whitham's result and Maslov's result

\begin{tabular}{lll}
\hline$\mu$ & \multicolumn{1}{c}{$B_{1}$} & $B_{3}$ \\
\hline 0 & 0.00 & 0.00 \\
0.5 & -0.18292683 & -0.22842144 \\
1 & -0.26315789 & -0.41225898 \\
1.5 & -0.28074866 & -0.54515265 \\
2 & -0.6315796 & -0.62393547 \\
2.5 & -0.22727273 & -0.64814813 \\
3 & -0.18292683 & -0.61873780 \\
3.5 & -0.13565892 & -0.53731328 \\
4 & -0.088495586 & -0.40575466 \\
4.5 & -0.043021033 & -0.22601149 \\
5 & $0.12417634 \times 10^{-7}$ & $0.74066669 \times 10^{-7}$ \\
\hline
\end{tabular}

For $\mu=5$, the table shows that $B_{3}$ is approximately six times that of $B_{1}$

\section{Conservation form of the equations on a shock manifold and the jump relations for the shock-shock}

In the last section we have derived two equations for the amplitude $\mu$ of the shock and for the angle $\Theta$ made by the normal to the shock front with the positive directions of the $x_{1}$-axis. As these equations are exact, it is natural and also interesting to analyse these equations further.

The SME (12) reduces to

$$
\hat{c} s / \hat{c} t+C|\nabla s|=0
$$

when the state ahead of the shock front is at rest.

For the subsequent part of the analysis we assume that $s$ is given by

$$
s\left(x_{x}, t\right)=t+\phi\left(x_{x}\right)
$$

then

$$
1+C\left(\phi_{x_{z}} \phi_{x_{x}}\right)^{1 / 2}=0 .
$$

Let us make a coordinate transformation from $\left(x_{\alpha}, t\right)$ to $\left(x_{\alpha}^{\prime}, s\right)$ given by

$$
x_{\alpha}^{\prime}=x_{x}, s=s\left(x_{x}, t\right)=t+\phi\left(x_{\alpha}\right) .
$$

In this coordinate system (56) becomes

$$
1+C\left(\phi_{x_{z}^{\prime}} \phi_{x_{x}^{\prime}}\right)^{1 / 2}=0 .
$$

The characteristic curves of (58) or shock rays are given by

$$
\mathrm{d} x_{x}^{\prime} / \mathrm{d} \tau=C \phi_{x_{x}^{\prime}} /\left(\phi_{x_{\beta}^{\prime}} \phi_{x_{\beta}^{\prime}}\right)^{1 / 2}=C N_{x},
$$

where $N_{1}=\cos \Theta, N_{2}=\sin \Theta$ are the components of the unit normal to the shock curve $\phi\left(x_{\alpha}^{\prime}\right)=$ constant.

The directional derivative operator along shock rays is

$$
\mathrm{d} / \mathrm{d} \tau=C N_{\alpha} \frac{\partial}{\partial x_{\alpha}^{\prime}}
$$


From (54) we get

$$
\frac{\mathrm{d} \Theta}{\mathrm{d} \tau}=\left(\sin \Theta \frac{\partial C}{\partial x_{1}^{\prime}}-\cos \Theta \frac{\partial C}{\partial x_{2}^{\prime}}\right)
$$

which with the help of $(60)$ can be written as

$$
C N_{\alpha} \frac{\partial \Theta}{\partial x_{\alpha}^{\prime}}-\left(\sin \Theta \frac{\partial C}{\partial x_{1}^{\prime}}-\cos \Theta \frac{\partial C}{\partial x_{2}^{\prime}}\right)=0
$$

or $\nabla^{\prime}\left(\mathbf{N}^{\prime} / C\right)=0^{\prime}$

on $\Omega$,

where $\nabla^{\prime}=\left(\frac{\partial}{\partial x_{1}^{\prime}}, \frac{\partial}{\partial x_{2}^{\prime}}\right)$ is the two-dimensional gradient in $\left(x_{\alpha}^{\prime}\right)$ coordinate system and $\mathbf{N}^{\prime}=(\sin \Theta,-\cos \Theta)$ is the unit vector tangential to the shock curve. Equation (62) is a conservation form of one of our shock dynamic equations.

Let us integrate the expression on the left side of (62) over the region $A B C D$ as shown in figure 1 where $A D, B C$ are two positions of shock fronts, $A B, C D$ are the shock rays. Then

$$
\begin{gathered}
\iint_{\operatorname{arca}} \nabla_{\mathrm{ABCD}} \cdot\left(\mathbf{N}^{\prime} / C\right)=0 \text { leads to } \\
\int_{(\mathrm{AB}+\mathrm{BC}+\mathrm{CD}+\mathrm{DA})} \frac{\mathrm{N}^{\prime} \cdot v}{C} \mathrm{~d} l=0
\end{gathered}
$$

$v$ being the exterior unit normal to the curve $\mathrm{ABCD}$. On $\mathrm{AD}$ and $\mathrm{BC} v \cdot \mathrm{N}^{\prime}=0$, on AB $v \cdot \mathbf{N}^{\prime}=1$ and on $C D v \cdot \mathbf{N}^{\prime}=-1$. Hence we get

$$
\int_{A B} \frac{\mathrm{d} l}{C}=\int_{C D} \frac{\mathrm{d} l}{C}
$$

Since $C$ is the velocity of the shock, the above equation implies that the shock front at any time $t$ can be obtained by moving a distance $C t$ along the rays starting from its initial position. Let us analyse (43) which can be written as

$$
\frac{\mathrm{d} \mu}{\mathrm{d} \tau}-B_{1} \frac{\partial \Theta}{\partial \eta}=B_{2} \frac{\partial \mu}{\partial N}
$$

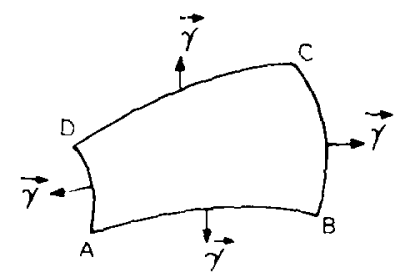

Figure 1. Elementary area whose boundaries are two positions of the shock front and two shock rays 
The right side expression in (63) represents the derivative of $\mu$ in the direction of normal to the shock front and accounts for the effect of flow from behind the shock. The flow behind the shock front depends on initial conditions and boundary conditions which can be chosen arbitrarily. Therefore, by choosing initial and boundary conditions in a proper way the term $B_{2} \frac{\partial \mu}{\partial N}$ can be made to assume any given value. Thus for the kinematics of a shock front this term should be treated as a known quantity.

Since

$$
\frac{\partial \Theta}{\partial \eta}=-\nabla \cdot \mathbf{N}=-\nabla^{\prime} \cdot \mathbf{N}^{\prime} \quad \text { and } \frac{\mathrm{d}}{\mathrm{d} \tau}=C N_{\alpha} \frac{\partial}{\partial x_{\alpha}^{\prime}}
$$

equation (63) becomes

$$
C N_{\alpha} \frac{\partial \mu}{\partial x_{\alpha}^{\prime}}+B_{1}(\mu) \frac{\partial N_{\alpha}}{\partial x_{\alpha}^{\prime}}=B_{2}(\mu) \frac{\partial \mu}{\partial N}
$$

We shall show that there exists a function $f(\mu)$ so that when $(64)$ is multiplied by it, it takes the form

$$
\nabla^{\prime} \cdot(K(\mu) \mathbf{N})=F\left(\mu, x_{1}^{\prime}, x_{2}^{\prime}\right),
$$

where $F\left(\mu, x_{1}^{\prime}, x_{2}^{\prime}\right)$ is a known function of $\mu, x_{1}, x_{2}$ and $K(\mu)$ is a function of $\mu$. Such a factor $f(\mu)$ has to satisfy the equations

$$
C(\mu) f(\mu)=K^{\prime}(\mu) \text { and } B_{1}(\mu) f(\mu)=K(\mu),
$$

which implies $\left(B_{1} f\right)^{\prime}=C f$ or $B_{1} \times f^{\prime}=f\left(C-B_{1}^{\prime}\right)$.

Thus $\quad f(\mu)=\left\{\exp \left[\int \frac{\left(C-B_{1}^{\prime}\right)}{B_{1}} \mathrm{~d} \mu\right]\right\}$

Hence $f(\mu)$ is determined upto a multiplicative constant. This constant can be taken to be one. Hence (64) can be written as

$$
\nabla^{\prime} \cdot(K(\mu) \mathbf{N})=F(\mu)
$$

Let us write explicitly the two conservation laws (62) and (65) on the shock manifold $\Omega$.

$$
\frac{\partial}{\partial x_{1}^{\prime}}\left(\frac{\sin \Theta}{C}\right)+\frac{\partial}{\partial x_{2}^{\prime}}\left(\frac{-\cos \Theta}{C}\right)=0,
$$

and

$$
\frac{\partial}{\partial x_{1}^{\prime}}(\mathrm{K} \cos \Theta)+\frac{\partial}{\partial x_{2}^{\prime}}(K \sin \Theta)=F(\mu) \text {. }
$$

The smooth solutions of the quasilinear equations derived from (66) and (67) develop into discontinuities in $\mu$ and $\Theta$ which are shock-shocks. From (66) and (67) we derive the jump condition across a shock-shock.

If $U=\mathrm{d} x_{2}^{\prime} / \mathrm{d} x_{1}^{\prime}$ is the slope of the shock-shock curve in $\left(x_{1}^{\prime}, x_{2}^{\prime}\right)$ plane then (66) and

$$
-U[K \cos \Theta]+[K \sin \Theta]=0
$$

and

$$
-U\left[\frac{\sin \Theta}{C}\right]+\left[\frac{-\cos \Theta}{C}\right]=0,
$$

where [ ] on any quantity represents its jump across the shock-shock. 
or

$$
U=\frac{\left(K_{2} \sin \Theta_{2}-K_{1} \sin \Theta_{1}\right)}{\left(K_{2} \cos \Theta_{2}-K_{1} \sin \Theta_{1}\right)}=\frac{\left(C_{2} \cos \Theta_{1}-C_{1} \cos \Theta_{2}\right)}{\left(C_{1} \sin \Theta_{2}-C_{2} \sin \Theta_{1}\right)},
$$

where $K_{2}=K\left(\mu_{2}\right), K_{1}=K\left(\mu_{1}\right), C_{2}=C\left(\mu_{2}\right), C_{1}=C\left(\mu_{1}\right)$ and subscripts 1 and 2 refer to the states on the two sides of shock-shock. Eliminating $U$ from these two we get

$$
\tan \left(\Theta_{1}-\Theta_{2}\right)=\frac{\left(K_{1}^{2}-K_{2}^{2}\right)^{1 / 2}\left(C_{1}^{2}-C_{2}^{2}\right)^{1 / 2}}{\left(K_{1} C_{2}+K_{2} C_{1}\right)}
$$

Whitham derived the jump relations across a shock-shock by considering the invariance of the distance traversed by the shock-shock. In contrast to this, we have derived these jump relations directly from the appropriate conservation laws which are also exact. We have given justification why the term $B_{2}(\partial \mu / \partial N)$ in $(65)$ should not contribute to the jump relations.

\section{References}

[1] Courant R and Friedrichs K O 1948 Supersonic flow and shock waves.

[2] Maslov V P 1980 Propagation of shock waves in the isentropic nonviscous gas. J. Sov. Maths. 13, 119-163

[3] Prasad P 1982 Kinematics of a multi-dimensional shock of arbitrary strength in an ideal gas. Acta Mech. $45163-176$

[4] Ramanathan T M, Prasad P and Ravindran R 1984 On the propagation of a weak shock front: theory and application. Acta Mech. 51 167-177

[5] Ramanathan T M, Prasad P and Ravindran R On the question of resolution of a hyperbolic caustic due to nonlinearity (sent for publication)

[6] Richtmyer R D and Morton K W 1967 Difference methods for initial value problems (New York: Interscience)

[7] Whitham G B 1957 A new approach to problems of shock dynamics Part I J. Fluid Mech. 2 146-171

[8] Whitham G B 1959 A new approach to problems of shock dynamics, Part II, Three-dimensional problems. J. Fluid Mech. $5369-386$ 